\title{
Perspective
}

PERSPECTIVE Actualité en histoire de l'art

2| 2007

La Grande-Bretagne/Période moderne

\section{Histoires de lièvres et de tortues : inventaires et guides d'architecture en Grande-Bretagne}

\section{Oliver Garnett}

\section{(2) OpenEdition}

1 Journals

\section{Édition électronique}

URL : http://journals.openedition.org/perspective/3818

DOI : 10.4000/perspective.3818

ISSN : 2269-7721

Éditeur

Institut national d'histoire de l'art

\section{Édition imprimée}

Date de publication : 30 juin 2007

Pagination : 309-314

ISSN : 1777-7852

\section{Référence électronique}

Oliver Garnett, « Histoires de lièvres et de tortues : inventaires et guides d'architecture en GrandeBretagne », Perspective [En ligne], 2 | 2007, mis en ligne le 31 mars 2018, consulté le 01 octobre 2020 URL : http://journals.openedition.org/perspective/3818; DOI : https://doi.org/10.4000/perspective. 3818

Ce document a été généré automatiquement le 1 octobre 2020. 


\title{
Histoires de lièvres et de tortues : inventaires et guides d'architecture en Grande-Bretagne
}

\author{
Oliver Garnett
}

\section{Les guides d'architecture Pevsner}

1 La réouverture, en 2007, du Royal Festival Hall à South Bank (Londres), après deux années d'une méticuleuse restauration, fournit une bonne occasion pour reconsidérer la fortune critique d'un monument qui est son exact contemporain, la collection The Buildings of England ${ }^{1}$ de Sir Nikolaus Pevsner. Cornwall, le premier des guides régionaux de Pevsner sur le patrimoine architectural anglais, était paru en 1951. Quand l'année suivante fut publié London except the cities of London and Westminster, le Royal Festival Hall illustrait la couverture, alors que le bâtiment n'était inauguré que depuis un an. Ce choix n'avait rien de surprenant de la part d'un fervent moderniste comme Pevsner, mais les louanges qu'il formulait à l'égard de cette nouvelle construction l'étaient plus. Il n'hésitait pas à en comparer l'espace intérieur à ceux des églises baroques d'Allemagne ou d'Autriche, rappelant ainsi au lecteur la perspective européenne qu'il avait adoptée pour ce travail. Durant vingt-deux ans, il dirigea et publia des volumes sur chaque comté anglais, la série s'achevant avec Staffordshire, en 1974. S'il ne put assumer seul les dernières parutions, cette entreprise reste néanmoins une extraordinaire performance physique et intellectuelle.

2 Tout comme le Royal Festival Hall, The Buildings of England ne fut pas exempt de critiques $^{2}$. John Harris, le plus virulent de ses contempteurs, pointait les nombreuses omissions ${ }^{3}$, dont Pevsner lui-même était d'ailleurs bien conscient: "Les premières éditions ne sont que des ballons d'essai; ce sont les secondes qui comptent $»^{4}$. On se souvient que David Watkin avait dénoncé chez Pevsner une tendance déterministe du type d'une Zeitgeschichte néohégélienne ${ }^{5}$, tout en n'étant pas avare de louanges pour la 
collection : «Personne depuis Ruskin n'a fait plus que Pevsner pour ouvrir les yeux de l'Anglais moyen sur son patrimoine architectural $»^{6}$.

3 The Buildings of England n'a jamais rapporté d'argent à son premier éditeur, Penguin Books, mais le soutien financier du Leverhulme Trust et, depuis 1964, du Buildings Books Trust a permis non seulement la publication des secondes éditions que Pevsner appelait de ses vœux mais aussi l'extension de la collection à l'Écosse, l'Irlande et le Pays de Galles. Depuis l'édition revue et corrigée du volume sur le Devon par Bridget Cherry en 1989, la collection est publiée dans un format plus grand pour faire place à de nouvelles entrées plus développées et à un index des commanditaires et des propriétaires (Pevsner avait été critiqué pour ne pas avoir pris suffisamment en compte l'aspect de la commande en architecture). Mais le volume Devon est à lui seul, sans même le glossaire, un livre de 976 pages, et, comme le reconnaissait B. Cherry ellemême, il ne s'agissait plus là d'un " livre de poche, tant du point de vue de sa taille que de son prix " (p. 14). Depuis 1998, la collection est connue sous l'appellation Pevsner architectural guides, faisant entrer ainsi son initiateur au panthéon des directeurs de collections éponymes du Xix ${ }^{e}$ siècle, aux côtés de Baedeker et de Murray.

En juillet 2002 s'est tenue une conférence au Victoria \& Albert Museum pour célébrer le centenaire de la naissance de Pevsner7 ${ }^{7}$. Les multiples projets qu'il avait menés à bien dans quantité de domaines, sans compter The Buildings of England, étaient au programme, et les conclusions auxquelles aboutirent les participants furent d'une grande variété - ce qui n'est sans doute pas pour étonner. L'un des fils rouges fut la façon ambiguë dont Pevsner fit sien «l'anglicité » [englishness]. Il faut se souvenir, à ce sujet, que The Buildings of England, qui aujourd'hui nous semble une collection tout ce qu'il y a de plus anglaise, s'inspirait à l'origine du Handbuch der deutschen Kunstdenkmäler de Georg Dehio (volumes parus de 1900 à 1913)².

5 Aujourd'hui les guides sont publiés par les éditions Yale University Press qui se sont massivement engagées dans les études sur l'art britannique depuis une vingtaine d'années. Elles continuent de publier environ deux volumes revus et corrigés par an, le dernier en date étant celui sur le comté d'Essex, par James Bettley. Les ouvrages sont à présent illustrés en couleurs, accompagnés de nombreux plans, et les titres se sont beaucoup diversifiés même si, et ce peut-être de façon inévitable, la patte si particulière de Pevsner et son style si original sont de moins en moins perceptibles. Mais la rigueur scientifique et l'attention portées aux descriptions détaillées restent toujours de mise.

6 L'approche de Pevsner a toujours mieux fonctionné pour certains domaines (comme les églises rurales) que pour d'autres (les grandes conurbations), mais cela ne s'est vraiment senti que lorsque les volumes ont augmenté en taille: celui sur le Grand Londres occupe à présent six tomes. L'une des solutions fut de reprendre les informations concernant tel ou tel bâtiment ou région spécifique dans une édition meilleur marché (les premiers volumes de la collection avaient été republiés en édition de poche) : c'est le cas des titres sur les docks de Londres (Elizabeth Williamson, Nikolaus Pevsner, London Docklands, Londres, 1998) et sur les églises de la City (Simon Bradley, Nikolaus Pevsner, London: The City Churches, Londres, 1998). Un autre choix, plus radical, fut de publier des guides totalement inédits consacrés aux grandes villes anglaises (le premier d'entre eux fut celui de Clare Hartwell, Manchester, Londres, 2001, puis parurent ensuite des volumes sur Bristol [2004], Liverpool [2004], Birmingham [2005] et Leeds [2005]). Mais le principe reste le même : les livres proposent des entrées consacrées aux principaux bâtiments publics ainsi que des propositions de promenades 
dans la ville et ses faubourgs ; le texte toutefois est plus narratif, et la maquette utilise les outils graphiques les plus récents, intégrant dans l'ensemble de l'ouvrage des encadrés, et des illustrations et cartes en couleur. Les «Pevsner » sont redevenus ce qu'ils étaient à l'origine: des guides maniables et pratiques - mais privés de leur dimension d'inventaire.

\section{Le Victoria County History ( $\mathrm{VCH})$}

7 Le $\mathrm{VCH}$ est une entreprise privée fondée en 1899 dont l'objectif était d'offrir une histoire régionale de l'Angleterre par comtés et par paroisses'. Après un début en fanfare, le projet fut sur le point de s'arrêter dans les années 1920. Il fut sauvé par l'Institute of Historical Research (IHR) de l'University of London qui l'héberge depuis lors, mettant à la disposition des rédacteurs des régions un centre d'informations bibliographique et scientifique. Plus de deux cents volumes ont paru à ce jour, mais le projet doit en compter le double.

8 Dès le premier titre, les descriptions architecturales des principaux bâtiments tenaient une grande place dans les volumes topographiques du $\mathrm{VCH}$, mais le travail d'inventaire du RCHME (voir ci-dessous), puis des guides Pevsner, conduisit le VCH à réduire cette ambition. Bien entendu, les premiers titres sont aujourd'hui obsolètes et l'on reprocha au $\mathrm{VCH}$ le caractère conservateur de sa politique éditoriale dont le but est de «maintenir des pratiques traditionnelles jusqu'au moment où elles se révèlent dépourvues de sens $\aleph^{10}$. Le texte est toujours dense et des notes de bas de page viennent garantir la validité de la moindre affirmation (les guides Pevsner proposent quant à eux un texte sans aucune note). Alors que la maquette extérieure de ces gros volumes rouges n'a pas beaucoup évolué, leur qualité scientifique s'est considérablement améliorée et le $\mathrm{VCH}$ a rendu un grand nombre des titres disponibles en ligne dans la section « British History Online » du site Internet de l'IHR (www.british-history.ac.uk), ce qui les rend bien plus facilement accessibles. Soutenu par le Heritage Lottery Fund, le $\mathrm{VCH}$ a lancé récemment un «England's Past for Everyone » qui prévoit d'offrir des informations concernant l'histoire des régions au public scolaire et aux amateurs éclairés sous une forme plus vulgarisée et interactive, et de publier d'autres courts volumes en format de poche. Le premier d'entre eux, Codford : wool and war in Wiltshire, va bientôt voir le jour; une étude sur le Hardwick Hall Estate (Derbyshire) suivra prochainement.

\section{La Royal Commission on the Historical Monuments of England (RCHME) et les répertoires d'édifices}

9 La RCHME fut mise en place par le gouvernement en 1908 afin de "procéder à un inventaire des monuments anciens et historiques d'Angleterre » : ce fut là sa principale mission durant les cinquante premières années de son existence ${ }^{11}$. À l'origine, ses experts ne prenaient en compte le patrimoine qu'antérieur à 1714 (fin de la dynastie des Stuart), aussi lorsque Pevsner rédigea ses guides sur Londres au début des années 1950, 75\% des bâtiments qu'il souhaitait traiter dans ses ouvrages n'étaient-ils pas décrits dans les volumes du RCHME correspondants. Malgré cette limite chronologique, la publication des inventaires régionaux du RCHME avança à pas de tortue. En 1979, il fut décidé de recentrer la publication sur des suppléments thématiques (la Royal 
Commission on the Ancient and Historical Monuments of Scotland, qui existe encore de nos jours, a mis un terme à la publication de ses inventaires régionaux en 1992). Durant les années 1980 et 1990, de nombreux titres parurent, sur des sujets allant des maisons de campagnes du Northamptonshire aux hospices anglais. Quand la RCHME fut intégrée à l'English Heritage en 1999, ce programme, malheureusement, fut arrêté, alors que les fruits de ces recherches continuèrent à être publiés en d'autres lieux ${ }^{12}$. Plus encourageant: le Survey of London ${ }^{13}$, qui survécut à la disparition de la RCHME, continue de publier son inventaire architectural des bâtiments de la capitale, paroisse par paroisse, bien qu'à un train de sénateur...: le quarante-cinquième et dernier volume paru, Knightsbridge, a vu le jour en 2003. L'English Heritage est en train de mettre en ligne les volumes les plus anciens (www.english-heritage.org.uk/server/ show/nav.9292).

Des inventaires nationaux de bâtiments historiques en Grande-Bretagne sont encore aujourd'hui publiés sous une forme traditionnelle. L'un des plus ambitieux est l'étude dirigée par Anthony Emery, trois volumes sur les grands manoirs du bas Moyen Âge en Angleterre et au Pays de Galles qui analysent les bâtiments un par un aussi scrupuleusement que le faisait la RCHME (Greater Medieval Houses of England and Wales, 1300-1500, Cambridge : 1, Northern England, 1996 ; 2, East Anglia, Central England, and Wales, 2000 ; 3, Southern England, 2006). La liste officielle des bâtiments importants (placés sous la responsabilité de l'English Heritage depuis 1995) a également généré des guides aussi utiles que celui de Jeremy Gould - un index par comtés des maisons de l'entre-deuxguerres au Royaume-Uni et en Irlande (Jeremy Gould, « Gazetteer of modern houses in the United Kingdom and the Republic of Ireland, 1919-1939», dans Journal of the Twentieth Century Society, 2, 1996, p.112-128; pour compléter la liste des maisons privées dessinées par des architectes après-guerre, voir Matthew Hardy, "List of architect-designed houses in England, 1945-1975 », dans Journal of the Twentieth Century Society, 4, 2000, p. 72-88).

11 Les guides sur des bâtiments historiques précis restent une source riche en informations, malgré les pressions commerciales qui tendent à les illustrer de plus en plus. En 2005, l'English Heritage réorganisa toute sa collection de guides de monuments, conservant les descriptions détaillées de la structure des bâtiments selon des critères scientifiques mais les présentant sous une maquette plus contemporaine même si elle reste tout de même assez austère. Le premier de ces guides fut celui de Roger White, Belsay Hall, Castle and Gardens, Londres, 2005. Le Cadw (l'équivalent de l'English Heritage au Pays de Galles) a adopté une approche semblable. L'association Historic Royal Palaces, quant à elle, a opté pour une orientation plus radicale, choisissant pour ses livres un format plus petit et une maquette inspirée des magazines de design contemporain, renonçant pour une grande part aux descriptions détaillées (Alan Bennett et al., Discover Kew Palace, Londres, 2006). Dans le même temps, elle a publié un grand nombre de solides volumes grand format d'histoires de son patrimoine (Susanne Groom, Lee Prosser, Kew Palace: The Official Illustrated History, Londres, 2006). Le National Trust a choisi une orientation intermédiaire, reprenant le traditionnel "tour du propriétaire ", mais redessinant la maquette de ses guides pour en faire des ouvrages de lecture plus aisée et plus souple. Il proposera aussi sous peu, via son site internet, plus d'informations spécialisées, comme la liste exhaustive des peintures des principales demeures qui relèvent de sa responsabilité. 


\section{Inventaires d'intérieurs}

12 Pevsner était peu favorable à la description du mobilier (hormis l'argenterie ecclésiastique de l'époque prévictorienne), notamment à cause des changements possibles de lieu de conservation; mais ces informations, portant à la fois sur le mobilier d'autrefois et sur celui d'aujourd'hui, sont évidemment cruciales pour comprendre l'histoire des intérieurs de maisons. La Walpole Society et la Furniture History Society continuent de publier des inventaires homologués d'intérieurs de bâtiments importants (Susan Jenkins, "An inventory of his Grace the Duke of Chandos's seat att Cannons taken June the 19th 1725' by John Gilbert ", dans Walpole Society, 67, 2005, p. 93-192, et Portrait of a Patron: The patronage and collecting of James Brydges, 1st Duke of Chandos, 1674-1744, Aldershot, 2007; (Santina M. Levey, Peter K. Thornton éd., Of Houshold Stuff: The 1601 inventories of Bess of Hardwick, dans Furniture history, 1971, Londres, 2001 ; Jonathan Marsden, "The Chastleton inventory of 1633 ", dans Furniture history, 36, 2000, p. 23-42). Sur ces documents les universitaires se sont longtemps appuyés pour comprendre l'apparence de l'habitat et ces publications nourrissent aussi le travail des chercheurs dans les domaines très en vogue des études sur les habitudes de consommation et des material studies ${ }^{14}$. Tessa Murdoch a récemment édité une collection non négligeable d'inventaires de maisons aristocratiques du XvIII ${ }^{\mathrm{e}}$ siècle (Tessa Murdoch éd., Noble Households: Eighteenth-century inventories of great English house: A tribute to John Cornforth, Cambridge, 2006). Espérons que d'autres publications du même type suivront.

D'insignes collections encore existantes méritent également d'être inventoriées et peu à peu ces inventaires voient le jour. Le National Trust a récemment publié des catalogues scientifiques des très fameuses collections d'argenterie de Dunham Massey (Cheshire) et de broderie du Hardwick Hall dans le Derbyshire (James Lomax, James Rothwell, Country House Silver from Dunham Massey, Swindon, 2006 ; Santina M. Levey, The Embroideries at Hardwick Hall: A catalogue, Swindon, 2007) ${ }^{15}$. Le projet le plus ambitieux est de loin le Public Catalogue Foundation qui publie, comté par comté, des catalogues concis mais très illustrés des collections britanniques de peintures à l'huile, à l'acrylique et a tempera, avec une rapidité et une détermination qui n'ont d'égale que celles des premiers titres des guides Pevsner ${ }^{16}$.

\section{NOTES}

1. Sur l'histoire de The Buildings of England, voir Bridget Cherry, The Buildings of England, Ireland, Scotland and Wales: A short history and bibliography, Londres, 1998 (Cambridge,1983) ; Simon Bradley, Bridget Cherry, The Buildings of England: A Celebration Compiled to Mark Fifty Years of the Pevsner Architectural Guides, Londres, 2001. 
2. Timothy Mowl, Stylistic Cold Wars: Betjeman versus Pevsner, Londres, 2000.

3. John Harris, No Voice from the Hall, Londres, 1998, p. 96.

4. Nikolaus Pevsner, Staffordshire, Harmondsworth, 1974, p. 18.

5. David Watkin, Morality and Architecture, Oxford, 1977, part 3, passim.

6. David Watkin, lettre au Times Literary Supplement, 3 mars 1978, p. 256.

7. Textes publiés dans Peter Draper éd., Reassessing Nikolaus Pevsner, Aldershot, 2004 ; compte rendu par Tim Benton dans Journal of Design History, 19/4, 2006, p. 357-360.

8. Le propos des manuels de G. Dehio était d'« être de petite taille, facilement transportable, que le plan du livre soit aussi clair que possible et que l'ouvrage soit aussi agréable à manier au bureau qu'en voyage » (Pevsner, 1974, cité n. 4, p. 8).

9. Sur l'histoire du VCH, voir R. B. Pugh éd., The Victoria History of the Counties of England: General introduction, Oxford, 1970, et également «From antiquarianism to professionalism: The progress of the Victoria county history ", dans Times Literary Supplement, 13 November 1970, p. 1327-1328 ; C. R. Elrington éd., The Victoria History of the Counties of England: General introduction: Supplement 1970-90, Oxford, 1990 ; C. R. J. Currie, C. P. Lewis éd., English County Histories: A guide, Stroud, 1994.

10. Elrington, 1990, cité n. 9, p. 4.

11. Sur l'histoire de la RCHME, voir Royal Commission on the Historical Monuments of England: A history and a final report, Swindon, 1998-1999, et Andrew Sargent, Royal Commission on the Historical Monuments of England, 1908-1998, (Transactions of the Ancient Monuments Society, 45), Londres, 2001, p. 57-80. Sur son équivalent écossais, voir J. G. Dunbar, The Royal Commission on the Ancient and Historical Monuments of Scotland, (Transactions of the Ancient Monuments Society, 36), Londres, 1992, p. 13-77.

12. Voir par exemple Nicholas Cooper, The Houses of the Gentry, 1480-1680, Londres, 1999 ; Kathryn Morrison, English Shops and Shopping: An Architectural History, Londres, 2003. Le patrimoine anglais a toutefois continué à être publié dans la collection de Christopher Stell Inventory of Nonconformist chapels and meeting houses, dont le dernier volume est paru en 2003. Pevsner avait reconnu que les bâtiments qui sortaient des normes architecturales traditionnelles étaient de fait sousreprésentés dans la première édition de ses guides (voir Pevsner, 1974, cité n. 4, p. 16).

13. Le Survey of London fut fondé en 1894 par l'architecte C. R. Ashbee et fut responsable, successivement, du London County Council, du Great London Council et de la RCHME. Sur l'histoire de cette institution, voir Hermione Hobhouse, Ninety Years of the Survey of London, (Transactions of the Ancient Monuments Society, 31), Londres, 1987, p. 25-47, et id., London Survey'd, Londres, 1994.

14. Pour une liste des inventaires de maisons de campagne anciennes, voir Maurice Howard, "Inventories, surveys and the history of great houses 1480-1640 ", dans Architectural History, 41, 1998, p. 14-29. Pour l'interprétation de ces inventaires, voir Maurice Howard, Edward Wilson, The Vyne: A Tudor House Revealed, Londres, 2003 (qui publie un inventaire de février 1541). Pour les inventaires et les material studies, voir John Styles, Amanda Vickery éd., Gender, Taste, and Material Culture in Britain and North America, 1700-1830, New Haven/Londres, 2006.

15. Giles Waterfield, dans son rapport de 2004 Attingham Trust Report - Opening doors: Learning in the historic environment (disponible sur http://www.openingdoorsreport.org.uk/), préconise une plus forte implication du National Trust dans les publications universitaires.

16. Voir www.thepcf.org.uk pour plus de détails. Le catalogue le plus récent couvre la Cornouaille et les îles Scilly. 
INDEX

Keywords : heritage, architecture history, inventory, monuments, publications, historiography Index géographique : Grande-Bretagne

Mots-clés : patrimoine, histoire de l'architecture, inventaire, monuments, publications, historiographie

Index chronologique : 1900

\section{AUTEURS}

\section{OLIVER GARNETT}

The National Trust 